\title{
On prime primitive roots
}

\author{
by
}

\section{Amora Nongkynrih (Madras)}

Notation. The letters $p, q$ and $l$ denote prime numbers. For a positive real number $H, N(H, p)$ denotes the number of primes $q \leq H$ which are primitive roots $(\bmod p) . N(\sigma, T, \chi)$ denotes the number of zeros of the Dirichlet $L$-function $L(s, \chi)$ in the rectangle $\sigma \leq \operatorname{Re} s \leq 1,-T \leq \operatorname{Im} s \leq T$.

For a given prime $p$, let

$$
F_{p}(s)=\prod_{\chi(\bmod p)} L(s, \chi) .
$$

For any positive integer $k, \log _{k} x$ is defined as follows: $\log _{1} x:=\log x$ and for $k \geq 2$, we inductively define $\log _{k} x=\log _{k-1} \log x$.

$[x]$ denotes the integral part of $x$.

1. Introduction. The purpose of this paper is to prove a result on the distribution of primitive roots, similar to one which appeared in a paper of Elliott [3], in which he obtained an asymptotic formula for $N(H, p)$, valid for "almost all" primes $p$. More precisely, he obtained the following (Theorem 1 of $[3])$ :

Let $\varepsilon$ and $B$ be arbitrary positive constants. Then there is a set of primes $E$, and a positive constant $F=F(\varepsilon, B)$, so that for all $p$ not in $E$ the estimate

$$
N(H, p)=\frac{\phi(p-1)}{p-1} \pi(H)\left\{1+O\left(\frac{1}{(\log H)^{B}}\right)\right\}
$$

holds uniformly for $H \geq \exp \left(F \log _{2} p \log _{3} p\right)$. Moreover, the sequence $E$ satisfies $E(x)=O\left(x^{\varepsilon}\right)$ for all large values of $x$.

In proving the result, Elliott had applied the first fundamental lemma (Lemma 4 of [3]), but there appears to be some discrepancy in the choice of the parameters in the application of the lemma. In this paper, we use a zero density estimate for $L$-functions and Brun's sieve to obtain an asymptotic formula for $N(H, p)$ which holds uniformly, for "almost all" primes $p$, in a 
larger range for $H$ than that stated in [3]. This arises as a special case of the asymptotic formula for $N(H, p)$ which holds for "almost all" $p$, in a wider range for $H$ at the expense of a weaker error term.

The theorem to be proved is the following:

Theorem 1.1. Let $\alpha$ be a real number satisfying $0<\alpha e^{1+\alpha} \leq 1$. Then for almost all primes $p$, the following statement is true:

Let $\alpha \geq c /\left(\log _{2} p\right)^{1 / 2}$, for a suitable constant $c$. Then, given $B>0$, there exists $C=C(B)$ such that whenever $H \geq \exp \left(\left(C \log _{2} p\right) / \alpha\right)$,

$$
N(H, p)=\frac{\phi(p-1)}{p-1} \pi(H)\left(1+O\left(\alpha^{B / \alpha}\right)\right) .
$$

Furthermore, the number of primes up to $Y$ for which (1) does not hold is

$$
O\left(\exp \left(\frac{G \log Y \log _{2} Y}{\log H}\right)\right)
$$

where $G$ is a constant.

Choosing $\alpha=\log _{4} p / \log _{3} p$ in Theorem 1.1, we get the following:

TheOREM 1.2. Let $\varepsilon$ and $B$ be arbitrary constants. Then for almost all primes $p$, the following holds:

$$
N(H, p)=\frac{\phi(p-1)}{p-1} \pi(H)\left(1+O\left(\frac{1}{(\log H)^{B}}\right)\right)
$$

whenever

$$
H \geq \exp \left(\frac{C \log _{2} p \log _{3} p}{\log _{4} p}\right),
$$

for some constant $C=C(\varepsilon, B)$. Furthermore, the number of primes up to $Y$ for which (2) does not hold is $O\left(Y^{\varepsilon}\right)$.

Corollary 1.3. If $E(Y)$ denotes the number of primes up to $Y$ for which (1) does not hold, then $E(Y)=O\left((\log Y)^{F}\right)$ when $H \geq Y^{\delta}$, for some $\delta$ and for some $F$, with $0<\delta<1$ and $F=F(\delta)$.

2. The exceptional primes. Call a prime $p$ an exceptional prime if (1) does not hold for $p$.

We need a lemma which was proved in a paper of Burgess and Elliott [1]. However, for our purposes, we require a different approach. We shall use Perron's formula to prove this lemma, and then apply a zero density estimate for $L$-functions. This will show that the number of exceptional primes is small.

To start with, we recall below the notation of Burgess and Elliott [1]: Let $\left\{\beta_{d, p}\right\}$ denote a double sequence of real numbers satisfying

$$
0 \leq \beta_{d, p} \leq 1 / \phi(d) \text {. }
$$


Define

$$
T_{p}=\sum_{\substack{d \mid p-1 \\ d>1}} \beta_{d, p} \sum_{\chi_{d}(\bmod p)}\left|\sum_{q \leq H} \chi_{d}(q)\right|
$$

where $\chi_{d}$ runs through the characters $(\bmod p)$ whose order is $d$. Let

$$
\varrho(p)=\sum_{\substack{d \mid p-1 \\ \beta_{d, p}>0}} 1
$$

Let $\lambda, R$ be positive real numbers, $Y \geq 3$. Define

$$
S_{1}=S_{1}(\lambda, R)=\left\{p \leq Y: \varrho(p)<R, T_{p}>\pi(H) / \lambda\right\} .
$$

LEMma 2.1. If $p$ is a prime for which $L(s, \chi)$ does not vanish for any character $\chi$ modulo $p$ (that is, $\left.F_{p}(s) \neq 0\right)$ in $\operatorname{Re} s>1-\varepsilon$, and $\varrho(p)<R$, then $T_{p}=O(\pi(H) / \lambda)$, provided

$$
\varepsilon \geq \max \left(\frac{4 \log R}{\log H}, \frac{2 \log \lambda}{\log H}, \frac{12 \log _{2} p}{\log H}\right) .
$$

Proof. Let $a$ and $T$ be real numbers such that $a>1$ and $T$ is sufficiently large. By Perron's formula, we have

$$
\sum_{n \leq H} \chi_{d}(n) \Lambda(n)=\frac{1}{2 \pi i} \int_{a-i T}^{a+i T}\left\{L^{\prime}\left(s, \chi_{d}\right) / L\left(s, \chi_{d}\right)\right\} \frac{H^{s}}{s} d s+O\left(\frac{H^{a} \log ^{2} p T}{T}\right)
$$

since $L^{\prime}\left(s, \chi_{d}\right) / L\left(s, \chi_{d}\right)=O\left(\log ^{2} p T\right)$ in $-1<\operatorname{Re} s \leq 2$, for a suitable choice of $\operatorname{Im} s=T$. (See, for example, [2].) Choose $a=1+1 / \log H$.

Since we are considering only primes $p$ with $F_{p}(s) \neq 0$ in $\operatorname{Re} s>1-\varepsilon$, moving the line of integration to $\operatorname{Re} s=1-\varepsilon$ gives

$$
\begin{aligned}
\sum_{n \leq H} \chi_{d}(n) \Lambda(n) & =\frac{1}{2 \pi i} \int_{1-\varepsilon-i T}^{1-\varepsilon+i T}\left\{L^{\prime}\left(s, \chi_{d}\right) / L\left(s, \chi_{d}\right)\right\} \frac{H^{s}}{s} d s+O\left(\frac{H \log ^{2} p T}{T}\right) \\
& =O\left(H^{1-\varepsilon} \log ^{2} p T \log T\right) .
\end{aligned}
$$

In particular, choosing $T=p$, we get

$$
\sum_{n \leq H} \chi_{d}(n) \Lambda(n)=O\left(H^{1-\varepsilon} \log ^{3} p\right) .
$$

Notice that

$$
\sum_{q<H} \chi_{d}(q) \log q=\sum_{n<H} \chi_{d}(n) \Lambda(n)+O\left(H^{1 / 2}\right)
$$

and that

$$
\sum_{n<m} \chi_{d}(n) \Lambda(n)=O\left(m^{1-\varepsilon} \log ^{3} p\right) \quad \text { for all } m<H .
$$


Thus, using Abel's identity and (3) it follows that

$$
\sum_{q<H} \chi_{d}(q)=O\left(H^{1-\varepsilon} \log ^{3} p\right) .
$$

Therefore,

$$
\begin{aligned}
T_{p} & =\sum_{\substack{d \mid p-1 \\
d>1}} \beta_{d, p} \sum_{\chi_{d}(\bmod p)}\left|\sum_{q<H} \chi_{d}(q)\right| \\
& \ll H^{1-\varepsilon} \log ^{3} p \sum_{\substack{d \mid p-1 \\
d>1}} \beta_{d, p} \phi(d)=H^{1-\varepsilon} \log ^{3} p\left(\sum_{\substack{d \mid p-1 \\
\beta_{d, p}>0}} 1\right) \\
& =H^{1-\varepsilon}\left(\log ^{3} p\right) R=H^{1-\varepsilon / 4} \lambda^{-1}\left(H^{-\varepsilon / 2} \lambda\right)\left(H^{-\varepsilon / 4} R\right) \log ^{3} p .
\end{aligned}
$$

Hence $T_{p}=O(\pi(H) / \lambda)$ whenever the following conditions hold: (i) $H^{-\varepsilon / 2} \lambda$ $<1$, (ii) $H^{-\varepsilon / 4} R<1$ and (iii) $\log ^{3} p<H^{\varepsilon / 4}$.

This completes the proof of the lemma.

We choose $R=(\log p)^{A}$, where $A$ is a sufficiently large constant, and $\lambda>R^{2}$; the value of $\lambda$ will be chosen in due course.

LEMMA 2.2 .

$$
\# S_{1} \ll \log ^{14} Y \exp \left(C \frac{\log \lambda \log Y}{\log H}\right) .
$$

Proof. Let $\varepsilon=2 \log \lambda / \log H$. Then

$$
\varepsilon \geq \max \left(\frac{4 \log R}{\log H}, \frac{2 \log \lambda}{\log H}, \frac{12 \log _{2} p}{\log H}\right) .
$$

Further, for any $p \in S_{1}, T_{p}>\pi(H) / \lambda$. Therefore, by Lemma 2.1, it follows that

$S_{1} \subseteq\left\{p \leq Y: F_{p}(s)=0\right.$ for some $s$ in the rectangle

$$
1-\varepsilon \leq \operatorname{Re} s \leq 1,-Y \leq \operatorname{Im} s \leq Y\} .
$$

Using the estimate

$$
\sum_{p \leq Y} \sum_{\chi}{ }^{\prime} N(\sigma, T, \chi) \ll\left(Y^{2} T\right)^{2(1-\sigma) / \sigma}(\log Y T)^{14}
$$

(here $\sum_{\chi}^{\prime}=$ the sum over all primitive characters $\chi$ modulo $p$ ) for $4 / 5 \leq$ $\sigma \leq 1$ (cf. Montgomery [5], p. 99), and also using our specific choice of $\varepsilon$, we see that

$$
\begin{aligned}
\sum_{Y<p \leq 2 Y} \sum_{\chi(\bmod p)} N(1-\varepsilon, Y, \chi) & \ll\left(Y^{3}\right)^{2 \varepsilon /(1-\varepsilon)}(\log Y)^{14} \\
& \ll Y^{(C \log \lambda) / \log H}(\log Y)^{14} .
\end{aligned}
$$

Hence $\# S_{1} \ll(\log Y)^{14} \exp (C \log \lambda \log Y / \log H)$, which proves the lemma. 
3. Derivation of the asymptotic formula. In this section, we consider only those primes for which $F_{p}(s) \neq 0$ in $\operatorname{Re} s>1-\varepsilon$, with $\varepsilon$ as chosen in Section 2. Given a prime $p$ with this property, we obtain an asymptotic formula for the number of prime primitive roots $(\bmod p)$ which are less than $H$.

Notice that if $d \mid p-1$, then

$$
\frac{1}{d} \sum_{\substack{\chi(\bmod p) \\ \operatorname{ord} \chi \mid d}} 1= \begin{cases}1 & \text { if } d \mid \text { ind } q, \\ 0 & \text { otherwise }\end{cases}
$$

where "ind $q$ " stands for the index of $q$ with respect to a fixed primitive root $\bmod p$.

Let $l$ denote a prime divisor of $p-1$. Then

$\#\{q \leq H: q$ is not a primitive $\operatorname{root}(\bmod p)\}$

$$
\leq \sum_{l \mid p-1} \frac{1}{l} \sum_{\text {ord } \chi \mid l} \sum_{q \leq H} \chi(q)=\pi(H) \sum_{l \mid p-1} \frac{1}{l}+\sum_{l \mid p-1} \frac{1}{l} \sum_{\chi_{l}} \sum_{q \leq H} \chi_{l}(q) .
$$

We break each sum into two parts: (i) $l \leq \log ^{2} p$, (ii) $l>\log ^{2} p$.

Lemma 3.1 below deals with the sum in (i) using Brun's sieve, and in Lemma 3.2 we estimate the sum in (ii) using Lemma 2.1. With notations as in [4], we state the following theorem, which is Brun's sieve in the form needed for our application (cf. [4], p. 57).

TheOREM 3.1. Assume that the following conditions hold:

(a)

$$
1 \leq \frac{1}{1-\omega(p) / p} \leq A_{1}
$$

for some suitable constant $A_{1} \geq 1$.

(b) For suitable constants $\kappa>0$ and $A_{2} \geq 1$,

$$
\sum_{w<p<z} \frac{\omega(p) \log p}{p} \leq \kappa \log \frac{z}{w}+A_{2}
$$

if $2 \leq w \leq z$.

(c) $\left|R_{d}\right| \leq \omega(d)$ if $\mu(d) \neq 0$ and $\omega(d) \neq 0$.

Let $\alpha$ be a real number satisfying $0<\alpha e^{1+\alpha} \leq 1$, and let $b$ be a positive integer. Then

$$
\begin{aligned}
S(\mathcal{A} ; \wp, z) \leq X W(z)\left\{1+2 \frac{\alpha^{2 b+1} e^{2 \alpha}}{1-\alpha^{2} e^{2+2 \alpha}}\right. & \left.\exp \left(\frac{(2 b+3) c_{1}}{\alpha \log z}\right)\right\} \\
& +O\left(z^{2 b+\left\{2.01 /\left(e^{2 \alpha / \kappa}-1\right)\right\}}\right)
\end{aligned}
$$


and

(6) $\begin{aligned} S(\mathcal{A} ; \wp, z) \geq X W(z)\left\{1-2 \frac{\alpha^{2 b} e^{2 \alpha}}{1-\alpha^{2} e^{2+2 \alpha}}\right. & \left.\exp \left(\frac{(2 b+2) c_{1}}{\alpha \log z}\right)\right\} \\ & +O\left(z^{2 b-1+\left\{2.01 /\left(e^{2 \alpha / \kappa}-1\right)\right\}}\right)\end{aligned}$

where

$$
c_{1}=\frac{A_{2}}{2}\left\{1+A_{1}\left(\kappa+\frac{A_{2}}{\log 2}\right)\right\} .
$$

Remark 1 . The constants implied by the use of the $O$-notation do not depend on $b$ and $\alpha$.

Remark 2. The replacement of the condition (c) of the theorem by the more general $\left|R_{d}\right| \leq L \omega(d)$ changes the theorem only to the extent of introducing a factor $L$ into the last error term in each of (5) and (6).

LEMMA 3.1 (Application of Brun's sieve). Let $p$ be a prime for which $F_{p}(s)$ is non-zero in $\operatorname{Re} s>1-(2 \log \lambda / \log H)$. Let $\mathcal{A}=\{\operatorname{ind} q: q \leq H\}$, $z=\log ^{2} p$, and $\wp=$ the set of all prime divisors $l$ of $p-1$. Then

$$
S(\mathcal{A} ; \wp, z)=\frac{\phi(p-1)}{p-1} \pi(H)\left(1+O\left(\alpha^{B / \alpha}\right)\right)
$$

where $\alpha$ is a real number satisfying $0<\alpha e^{1+\alpha} \leq 1, \alpha \gg 1 /(\log z)^{1 / 2}$, and $B$ is a constant.

Proof. With these choices of $\mathcal{A}, \wp$ and $z$, it follows that

$$
\omega(p)=1 \quad \text { if } p \in \wp, \quad X=\pi(H), \quad \kappa=1,
$$

and

$$
W(z)=\prod_{\substack{q \mid p-1 \\ q<z}}\left(1-\frac{1}{q}\right)
$$

We see that

$$
\#\{q \leq H: d \mid \text { ind } q, d \mid p-1\}=\frac{1}{d} \sum_{q \leq H} \sum_{\substack{\chi(\bmod p) \\ \text { ord } \chi \mid d}} \chi(q) .
$$

Hence,

$$
\begin{aligned}
\left|\mathcal{A}_{d}\right| & =\frac{1}{d} \sum_{\substack{\chi(\bmod p) \\
\text { ord } \chi \mid d}} \sum_{q \leq H} \chi(q)=\frac{1}{d} \pi(H)+\frac{1}{d} \sum_{\substack{\chi \neq \chi_{0} \\
\text { ord } \chi \mid d}} \sum_{q \leq H} \chi(q) \\
& =\frac{1}{d} \pi(H)+\frac{1}{d} \sum_{\substack{t \mid d \\
t>1}} \sum_{\chi t} \sum_{q \leq H} \chi_{t}(q)
\end{aligned}
$$


where $\chi_{t}$ runs through characters of order $t$. Therefore,

$$
R_{d}=\frac{1}{d} \sum_{\substack{t \mid d \\ t>1}} \sum_{\chi_{t}} \sum_{q \leq H} \chi_{t}(q) .
$$

Using (4), we get

$$
\begin{aligned}
\left|R_{d}\right| & \ll \frac{1}{d} \sum_{t \mid d} \sum_{\chi_{t}}\left|\sum_{q \leq H} \chi_{t}(q)\right| \ll\left(\frac{1}{d} \sum_{t \mid d} \sum_{\chi_{t}} 1\right) H^{1-\varepsilon} \log ^{3} p \\
& \ll\left(\frac{1}{d} \sum_{t \mid d} \phi(t)\right) H^{1-\varepsilon} \log ^{3} p=H^{1-\varepsilon} \log ^{3} p \ll \pi(H) / \lambda .
\end{aligned}
$$

The last step follows as in the proof of Lemma 2.1. We take $b=[1 / \alpha]$ in Theorem 3.1, and Brun's sieve then gives

$$
\begin{array}{r}
S(\mathcal{A} ; \wp, z) \leq \pi(H) W(z)\left\{1+2 \frac{\alpha^{2 b+1} e^{2 \alpha}}{1-\alpha^{2} e^{2+2 \alpha}} \exp \left(\frac{(2 b+3) c_{1}}{\alpha \log z}\right)\right\} \\
+O\left(\frac{\pi(H)}{\lambda} z^{2 b+\left\{2.01 /\left(e^{2 \alpha}-1\right)\right\}}\right)
\end{array}
$$

and

(8) $S(\mathcal{A} ; \wp, z) \geq \pi(H) W(z)\left\{1-2 \frac{\alpha^{2 b} e^{2 \alpha}}{1-\alpha^{2} e^{2+2 \alpha}} \exp \left(\frac{(2 b+2) c_{1}}{\alpha \log z}\right)\right\}$

$$
+O\left(\frac{\pi(H)}{\lambda} z^{2 b-1+\left\{2.01 /\left(e^{2 \alpha}-1\right)\right\}}\right)
$$

with

$$
\begin{aligned}
W(z) & =\prod_{q \mid p-1}\left(1-\frac{1}{q}\right) \prod_{\substack{q \mid p-1 \\
q \geq z}}\left(1-\frac{1}{q}\right)^{-1} \\
& =\frac{\phi(p-1)}{p-1}\left(1+O\left(\frac{1}{\log p \log _{2} p}\right)\right) .
\end{aligned}
$$

With our choice of $b$, we now estimate the error terms in (7). Similar estimates can be obtained for the inequality (8). The estimate for the first error term is

$$
\frac{\alpha^{2 b+1} e^{2 \alpha}}{1-\alpha^{2} e^{2+2 \alpha}} \exp \frac{(2 b+3) c_{1}}{\alpha \log z} \ll \alpha^{B / \alpha}
$$

whenever $\alpha^{2} \gg 1 / \log z$. Since $\alpha$ is small, the last $O$-term satisfies

$$
\frac{\pi(H)}{\lambda} \exp \left(\left(2 b+\left\{2.01 /\left(e^{2 \alpha}-1\right)\right\}\right) \log z\right) \ll \frac{\pi(H)}{\lambda} z^{B^{\prime} / \alpha}
$$

for a constant $B^{\prime}$. We choose $\lambda>z^{B^{\prime} / \alpha}=(\log p)^{2 B^{\prime} / \alpha}$. For our purposes, we take $\lambda$ to satisfy $\log \lambda=\left(C^{\prime} \log _{2} p\right) / \alpha$, for a sufficiently large constant $C^{\prime}$. 
Using the estimates in (7) and (8), it follows that

$$
\begin{aligned}
S(\mathcal{A} ; \wp, z)=\frac{\phi(p-1)}{p-1} \pi(H)\left(1+O\left(\frac{1}{\log p \log _{2} p}\right)\right) & \left(1+O\left(\alpha^{B / \alpha}\right)\right) \\
& +O\left(\frac{\pi(H) z^{B^{\prime} / \alpha}}{\lambda}\right) .
\end{aligned}
$$

Therefore, we get

$$
S(\mathcal{A} ; \wp, z)=\frac{\phi(p-1)}{p-1} \pi(H)\left(1+O\left(\alpha^{B / \alpha}\right)\right),
$$

which proves the lemma.

We now consider the sum in (ii).

LEMMA 3.2. Let

$$
L=\sum_{l>\log ^{2} p} \frac{1}{l}\left(\pi(H)+\sum_{\chi_{l}} \sum_{q \leq H} \chi_{l}(q)\right) .
$$

Then $L=O(\pi(H) / \log p)$.

Proof.

$$
L=\pi(H) \sum_{l>\log ^{2} p} \frac{1}{l}+\sum_{l>\log ^{2} p} \frac{1}{l} \sum_{\chi_{l}} \sum_{q \leq H} \chi_{l}(q) .
$$

Then

$$
|L| \leq \frac{\pi(H)}{\log p}+\sum_{l>\log ^{2} p} \frac{1}{l} \sum_{\chi_{l}}\left|\sum_{q \leq H} \chi_{l}(q)\right| \ll \frac{\pi(H)}{\log p}+\frac{\pi(H)}{\lambda},
$$

applying Lemma 2.1 to the second sum on the right with

$$
\beta_{l, p}= \begin{cases}1 / l & \text { if } l \mid p-1, l>\log ^{2} p, \\ 0 & \text { otherwise. }\end{cases}
$$

Therefore, $L=O(\pi(H) / \log p)$.

Proof of Theorem 1.1. Lemmas 3.1 and 3.2 imply that for almost all primes $p$,

$$
N(H, p)=\frac{\phi(p-1)}{p-1} \pi(H)\left(1+O\left(\alpha^{B / \alpha}\right)\right)
$$

where $\alpha \gg 1 /\left(\log _{2} p\right)^{1 / 2}$ and whenever $H \geq \exp \left(\left(C \log _{2} p\right) / \alpha\right)$ for some constant $C=C(B)$. Lemma 2.2 shows that the number of exceptional primes up to $Y$ is

$$
\ll(\log Y)^{14} \exp \left(\frac{C \log Y \log _{2} Y}{\alpha \log H}\right) .
$$

This completes the proof of Theorem 1.1. 
Acknowledgements. This paper is the result of a suggestion made by Professor M. Ram Murty. I would like to thank him and Professor R. Balasubramanian for constant guidance and encouragement.

\section{References}

[1] D. A. Burgess and P. D. T. A. Elliott, On the average value of the least primitive root, Mathematika 15 (1968), 39-50.

[2] H. Davenport, Multiplicative Number Theory, 2nd ed., Graduate Texts in Math. 74, Springer, New York, 1980.

[3] P. D. T. A. Elliott, The distribution of primitive roots, Canad. J. Math. 21 (1969), $822-841$

[4] H. Halberstam and H.-E. Richert, Sieve Methods, Academic Press, London, 1974.

[5] H. L. Montgomery, Topics in Multiplicative Number Theory, Lecture Notes in Math. 227, Springer, Berlin, 1971.

THE INSTITUTE OF MATHEMATICAL SCIENCES

C.I.T. CAMPUS

MADRAS 600113, INDIA

E-mail: AMORA@IMSC.ERNET.IN

Received on 10.11.1993

and in revised form on 13.5.1994 